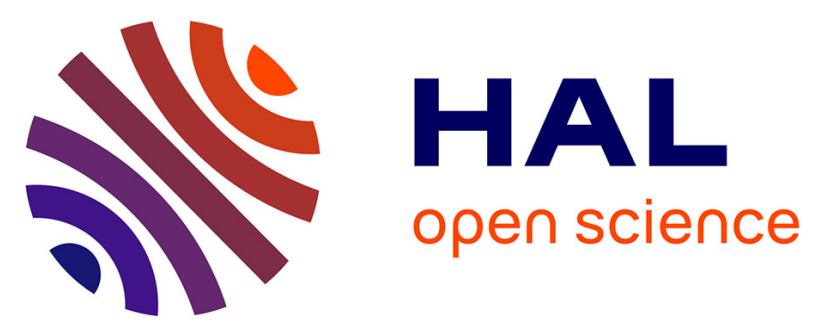

\title{
Results from prototypes of environmental and health alarm devices based on gaseous detectors operating in air in counting mode
}

Georges Charpak, Patrick Benaben, Philippe Breuil, Paolo Martinengo, Eugenio Nappi, Vladimir Peskov

\section{To cite this version:}

Georges Charpak, Patrick Benaben, Philippe Breuil, Paolo Martinengo, Eugenio Nappi, et al.. Results from prototypes of environmental and health alarm devices based on gaseous detectors operating in air in counting mode. Nuclear Instruments and Methods in Physics Research Section A: Accelerators, Spectrometers, Detectors and Associated Equipment, 2011, 628 (1), pp.197-189. 10.1016/j.nima.2010.06.313 . hal-00560493

\section{HAL Id: hal-00560493 https://hal.science/hal-00560493}

Submitted on 31 Jan 2011

HAL is a multi-disciplinary open access archive for the deposit and dissemination of scientific research documents, whether they are published or not. The documents may come from teaching and research institutions in France or abroad, or from public or private research centers.
L'archive ouverte pluridisciplinaire HAL, est destinée au dépôt et à la diffusion de documents scientifiques de niveau recherche, publiés ou non, émanant des établissements d'enseignement et de recherche français ou étrangers, des laboratoires publics ou privés. 


\title{
Results from prototypes of environmental and health alarm devices based on gaseous detectors operating in air in counting mode
}

\author{
Georges CharpaK(1), Patrick Benaben(1), Philippe ${ }^{(1)}$ Breuil $^{(2)}$, Paolo \\ MARTINENGO(3), EUGÉNIO NAPPI(4), VLADIMIR PESKOV ${ }^{(3) *}$
}

(1) Ecole Nationale Supérieure des Mines de Saint Etienne ; Centre CMP; Département PS2; 158 Cours Fauriel, 42023 Saint-Étienne Cedex 2, France

(2) Ecole Nationale Supérieure des Mines de Saint Etienne ; Centre SPIN ; Département PRESSIC ; LPMG-UMR CNRS 5148 ; 158 Cours Fauriel, 42023 Saint-Étienne Cedex 2, France

(3) CERN CH-1211, Genève 23, Suisse

(4) INFR (Istituto Nazionale di Fisica Nuclear), Via E. Orabona $N^{\circ} 4$, 70126, Bari, Italie

\begin{abstract}
We have developed and successfully tested two prototypes of detectors of dangerous gases based on wire-type counters operating in air in avalanche mode: one is for radon (Rn) detection whereas the other one is for the detection of gases with an ionization potential less than the air components. Due to the operation in pulse counting mode these prototypes have sensitivities comparable to (in the case of the Rn detector) or much higher than (in the case of the detector for low ionization gases) the best commercial devices currently available on the market. We believe that due to their high sensitivity, simplicity and low cost such new detectors will find massive applications. One of them, discussed in this paper, could be the online monitoring of $\mathrm{Rn}$ for the prediction of earthquakes.
\end{abstract}

Keywords:

Gaseous detectors; Rn detection; Detection of dangerous gases

\section{Introduction}

Environmental alarm, monitoring and health safety devices operating in air in ionization mode are commercially available: for example smoke detectors, sensors of dangerous gases, medical X-ray devices and charged particle dosimeters. The sensitivity of these detectors is mainly determined by the sensitivity of compact pico-amperemeters mounted inside the devices (typically $\sim \mathrm{pA}$ ). In this work, we investigated the possibility to exploit the gaseous detectors in air in avalanche mode. In this case the gaseous detector will be a naturally integrated preamplifier for the electronic circuit, allowing to not only make the sensor simpler and cheaper, but in some cases also significantly increasing its sensitivity. In the following the first promising results obtained within this approach are presented.

\footnotetext{
${ }^{*}$ Corresponding author: Vladimir.peskov@cern.ch
} 
Nuclear Instruments and Methods in Physics Research Section A: Accelerators, Spectrometers, Detectors and Associated Equipment, 2011, 628(1), 187-189, Proceedings of the $12^{\text {th }}$ International Vienna Conference on Instrumentation,

doi:10.1016/j.nima.2010.06.313

\section{Results}

\section{II.1. Operation of gaseous detectors in air}

It is well known that air is not a good quenching gas, and as a consequence of this the traditional gaseous detectors (single-wire and parallel-plate) suffer from instabilities, especially from photon feedback [1]. For this reason, we have studied in our recent work [2] the operation in air of hole-type detectors in which, due to the avalanche confinement in the holes, the photon feedback is strongly reduced. Indeed, an exceptionally high gas gain could be achieved with these hole-type detectors; however, at air humidity above $70 \%$ they become noisy [2], due to the charge leak across the hole walls.

In this work, we investigated the feasibility to use wire-type counters instead of the hole-type detectors. The main interest of using a wire detector is that there is practically no limit in the size of the dielectric interface between the cathode cylinder and the anode wire, thus allowing the implementation of rectangular grooves [3] to suppress surface leakage due to humidity. Moreover, as was shown in [3], geometrical optimization of the wire detectors can significantly reduce the photon feedback, and they become capable of operating stably at gas gains of $10^{3}-10^{4}$. We used such optimized wire counters in several applications [3] and [4]. Below we will describe two of them: Rn detection and detection of some dangerous low ionization potential (LIP) gases in air.

\section{II.2. Radon detector}

It is well known that $\mathrm{Rn}$ gas from the environment, accumulating in buildings, especially in the basements, represents a significant threat to human health. Our work, however, was not focused on health safety (which is quite well covered with the existing detectors), but on the on-line detection of $\mathrm{Rn}$ as a possible precursor of a forthcoming earthquake. Indeed, some recent studies have shown the possibility to correlate rapid changes of the $\mathrm{Rn}$ concentration in soil or in groundwater to the early prediction of earthquakes (see [3] and references therein). In order to verify such observations on a more solid statistical ground it is needed to create a wide network of cheap, compact and high sensitivity Rn detectors, deployed in key points where the earthquake may potentially occur. The existing excellent commercial detectors are too expensive to be employed in large numbers.

Two types of detectors oriented towards this particular application were developed and tested in this work: a simple single-wire counter (SWC), extensively used for basic studies, and a more sophisticated detector, a multiwire proportional counter (MWPC).

The schematic drawing of the SWC is shown in Figure 1, its cathode cylinder is $60 \mathrm{~mm}$ in diameter, and the anode wire is $100 \mu \mathrm{m}$ in diameter. During the measurements a positive HV was applied to the anode wire whereas the outer cylinder was grounded.

The signals from the anode wire were detected by a custom made charge-sensitive amplifier able to deal with long-rise-time pulses (due to the long drift time of the negative ions), and if necessary they were in addition amplified by a research amplifier. In most of the measurements the signals were analyzed and displayed by a Labview program.

In Figure 2 a schematic drawing of the MWPC is shown. The outer cylinder, $40 \mathrm{~mm}$ in diameter and $50 \mathrm{~cm}$ long, was made of stainless steel (SS) or of a stainless steel mesh with anode and cathode wires inside, stretched parallel to the cylinder axis in a hexagonal arrangement. This design was actually an exact copy of the detector described in [5], and the same technology was used for the manufacturing. However for each anode wire, protective grooves (to minimize the charge leak across the surface) were implemented on both ends, at the top and the bottom dielectric flanges. In the measurements with alpha particles, only events with two or more wires producing signals within a gate of a few $\mu$ s have been accepted and analyzed.

As an example the signals, produced by a ${ }^{241} \mathrm{Am}$ source when the detector was filled with ambient air, are shown in Figure 3, and the mean amplitudes of the signals induced by alpha 
Nuclear Instruments and Methods in Physics Research Section A: Accelerators, Spectrometers, Detectors and Associated Equipment, 2011, 628(1), 187-189, Proceedings of the $12^{\text {th }}$ International Vienna Conference on Instrumentation, doi:10.1016/j.nima.2010.06.313

particles are depicted in Figure 4 as a function of the applied voltage, for different diameters of the anode wire. Both the SWC and the MWPC have a broad counting plateau [3] allowing to perform reliable measurements at various atmospheric conditions. The main results obtained with our air-filled detectors are summarized in Table 1, where the minimum detector activity (MDA) measured during different time intervals is reported. For comparison, the MDA for one of the best commercial Rn detectors, the Atmos [3], is also presented in the same table.

As can be seen from this table, the MDA of the MWPC is as low as the MDA of the best commercial detector. With our detectors we could easily detect small Rn traces in air (see also [3] for more details).

\section{II.3. Detectors for gases with a low ionization potential (LIP)}

Commercial photoionization detectors are working on the principle of ionizing LIP gases (benzene, toluene and others) by monochromatic UV radiation emitted from a lamp:

$M_{2}+h v=M_{2}^{+}+e^{-}$

By measuring the photocurrent I from the ionization chamber, the concentration $\mathrm{n}$ of the dangerous gases can be determined solving the following equation:

$$
I=N Q^{L} \int_{0} \exp (-K(d+x))\{\exp (-\sigma n x) d x / \sigma n\}
$$

where $N$ is the number of photons emitted per second by the lamp placed at a distance $d$ from the ionization chamber, $Q$ is the quantum efficiency of the LIP gas, $L$ is the length of the ionization chamber, $K$ is the UV light absorption coefficient in air, and $\sigma$ is the photo absorption cross-section of the LIP gas at the given UV wavelength. For the sake of simplicity, let us consider a particular case of a very low concentration of a LIP gas, and a UV wavelength at which the absorption in air is small, then $n=I / N Q n \sigma L$. Typically, the lowest concentration of the LIP gases which can be reliably detected with commercial photoionization sensors is in the range of 10-100 ppm. In paper [6] we already described a prototype of a photoionization detector for dangerous gases based on hole-type amplifiers which, due to the gas multiplication, allows to increase the sensitivity. In this work, we investigated if SWCs can be used instead of hole-type detectors, what may open up the possibility to exploit them in a humid atmosphere.

The experimental setup used for these studies was basically the same as described in [6]. The small modifications were: the introduction of an additional large-volume buffer gas chamber, allowing for the preparation of the gas mixtures with very low concentrations of LIP gases, and the possibility of using a pulsed Ar lamp inside the test chamber. The measurements were performed either in current mode (continuous UV source combined with a compact SWC operating with a lower gas gain) or in pulse mode (pulsed lamp combined with SWC operating in pulse mode), see [Figure 5] and [Figure 6]. In the case of current measurements and small values of $n$ and $K$, the concentration of the LIP gases can be calculated from the following formula: $n=I / A N Q n \sigma L$. As can be seen, for the given fixed lamp intensity the detection sensitivity is increased by a factor A.

In the case of pulse measurements, the integrated avalanche charge per lamp pulse emitting $N_{\mathrm{p}}$ photons is: $\Delta q=N_{\mathrm{p}} \sigma n L Q A$. The main advantage of the pulse mode is the easy way of calibrating the gas gain: for example using the radioactive sources ${ }^{241} \mathrm{Am}$ (see Figure 3) and 55Fe [3]. In Figure 6, as an example, are shown the signal amplitudes vs. the voltage, for the SWC operating in pulse mode and detecting various concentrations of benzene vapors. As can be seen, a benzene concentration as low as $0.03 \mathrm{ppm}$ could be detected in air, what is 10-100 times better than the typical sensitivity of commercial photoionization detectors. 
Nuclear Instruments and Methods in Physics Research Section A: Accelerators, Spectrometers, Detectors and Associated Equipment, 2011, 628(1), 187-189, Proceedings of the $12^{\text {th }}$ International Vienna Conference on Instrumentation,

doi:10.1016/j.nima.2010.06.313

\section{References}

[1] H. Raether, Electron Avalanches and Breakdown in Gases, Butterworths, London (1964).

[2] G. Charpak et al., JINST 3 (2008), p. Po2oo6.

[3] G. Charpak et al., arXiv:1002.4732, 2010.

[4] G. Charpak et al., JINST 4 (2009), p. P12007.

[5] R. Bouclier et al., Nucl. Instr. and Meth. A 252 (1986), p. 373.

[6] G. Charpak et al., IEEE Trans. Nucl. Sci. NS55 (2008), p. 1657.

\section{Figures}

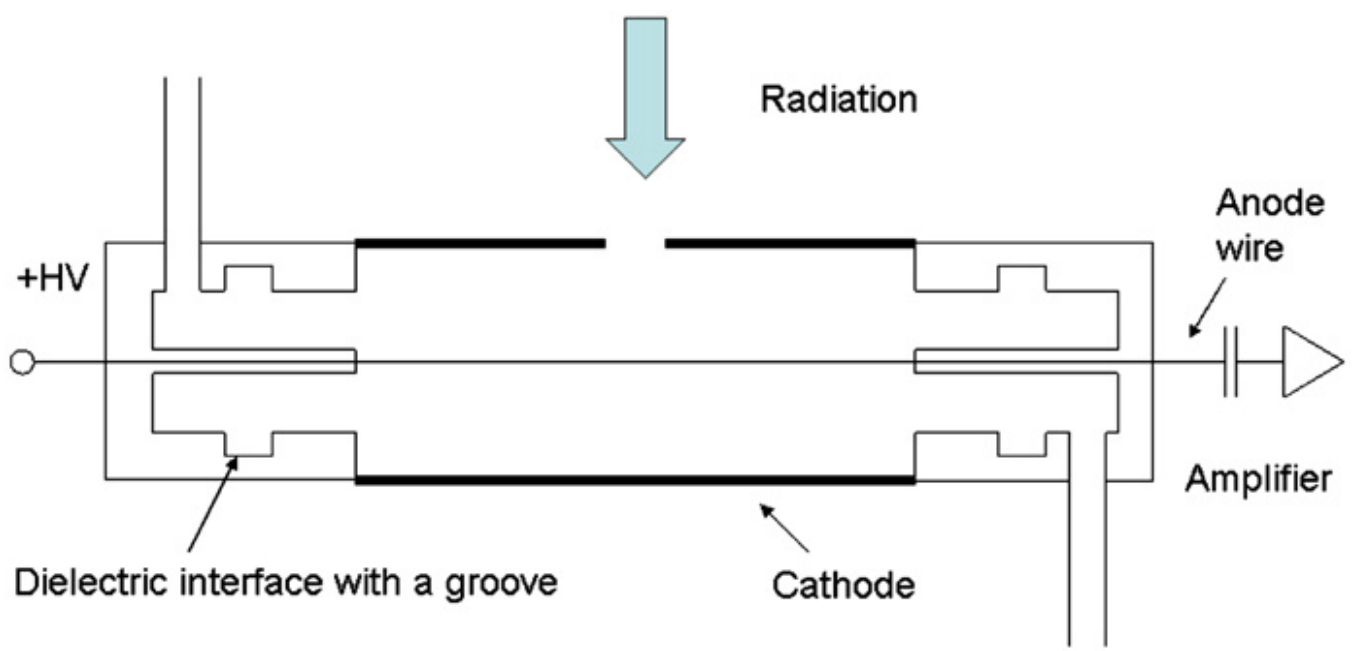

Figure 1: Schematic drawing of the single-wire counter operating in humid ambient air at high gains.

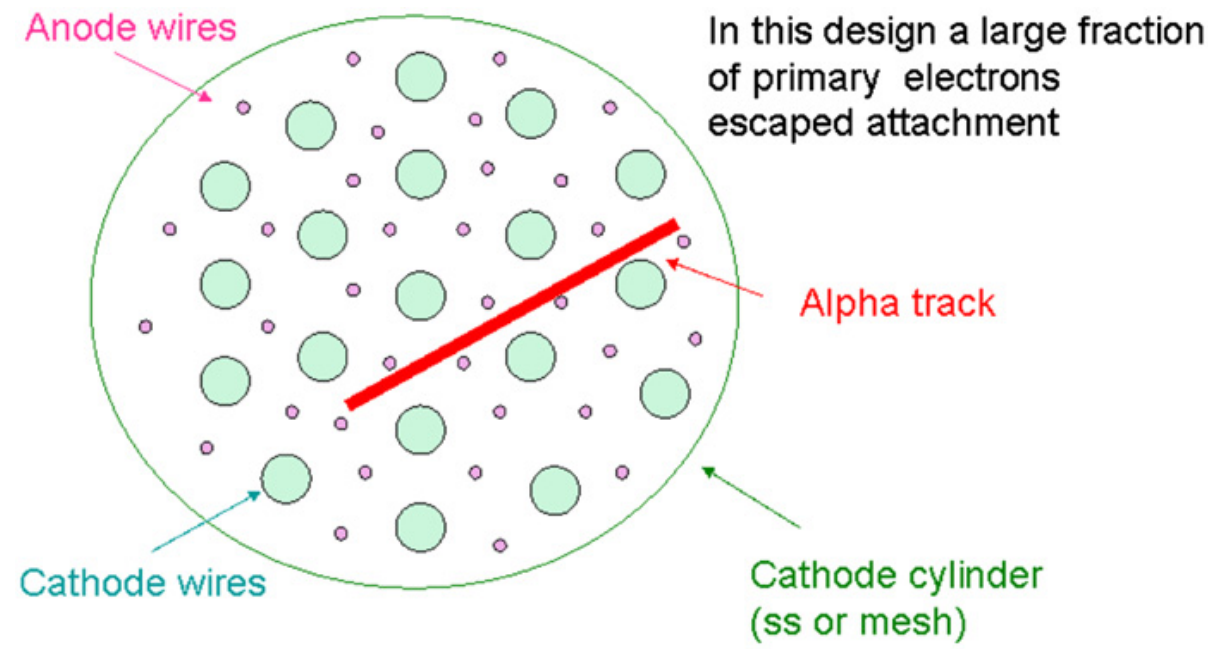

Figure 2: Schematic cross-section of the MWPC detector. 
Nuclear Instruments and Methods in Physics Research Section A: Accelerators, Spectrometers, Detectors and Associated Equipment, 2011, 628(1), 187-189, Proceedings of the $12^{\text {th }}$ International Vienna Conference on Instrumentation,

doi:10.1016/j.nima.2010.06.313

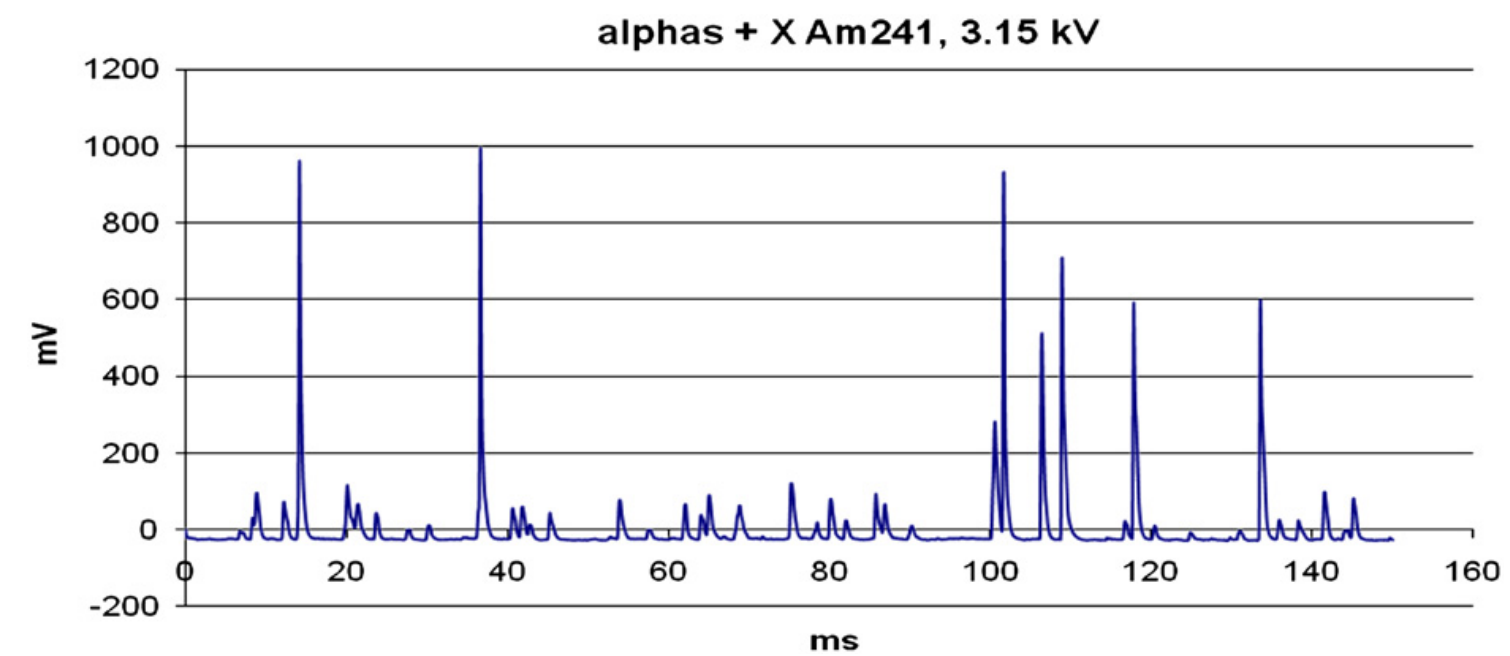

Figure 3: Typical signals measured with a SWC operating in air and irradiated with $a^{241} \mathrm{Am}$ source. The large pulses were produced by alpha particles whereas the small ones were caused by $60 \mathrm{keV}$

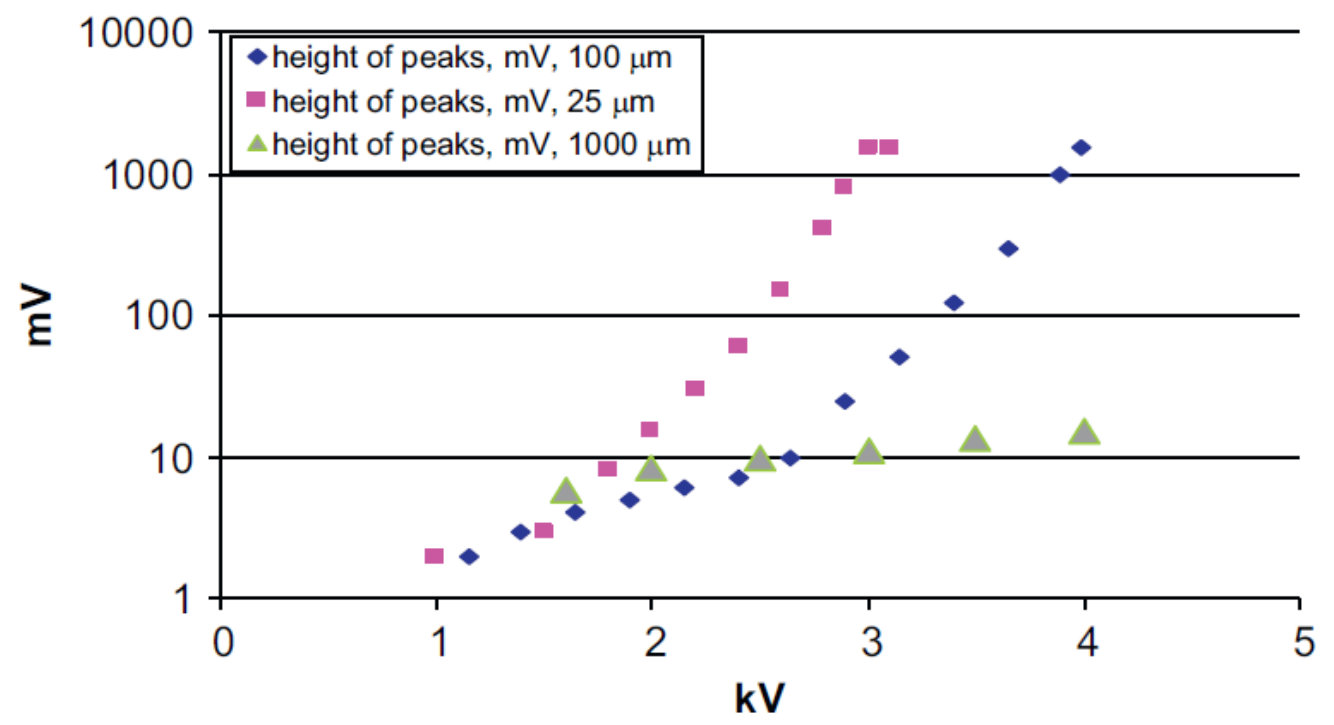

Figure 4: Mean signal amplitude produced by alpha particles vs. the voltage applied to detectors having a cathode diameter of $60 \mathrm{~mm}$ and different diameters for the anode wires. 
Nuclear Instruments and Methods in Physics Research Section A: Accelerators, Spectrometers, Detectors and Associated Equipment, 2011, 628(1), 187-189, Proceedings of the $12^{\text {th }}$ International Vienna Conference on Instrumentation,

doi:10.1016/j.nima.2010.06.313

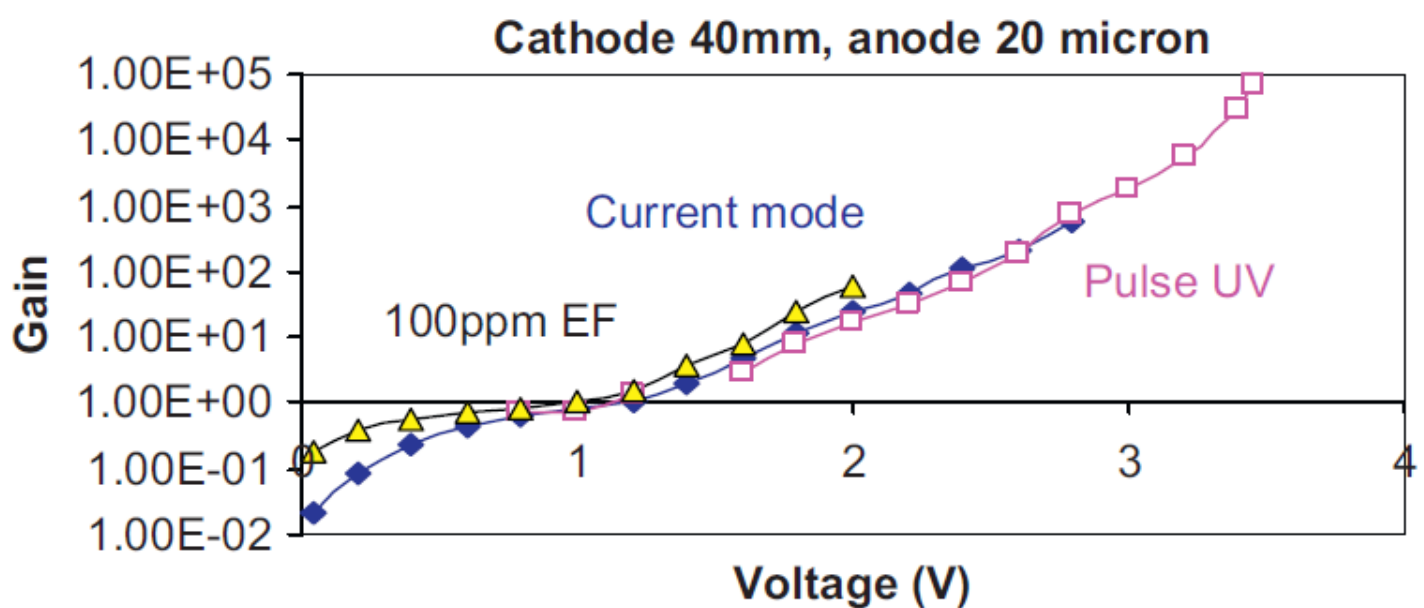

Figure 5: Gain vs. applied voltage curves for the SWC operating in current mode (open triangles and filled rhombuses) and in pulse mode (open squares). The triangles represent measurements with $100 \mathrm{ppm}$ ethylferovene vapors (the UV light does not hit the SWC cathode). The rhombuses and squares are the gain calibration curves measured using a surface photoeffect from the cathode (for the purpose of these particular measurements the cathode was exposed to the lamp light).

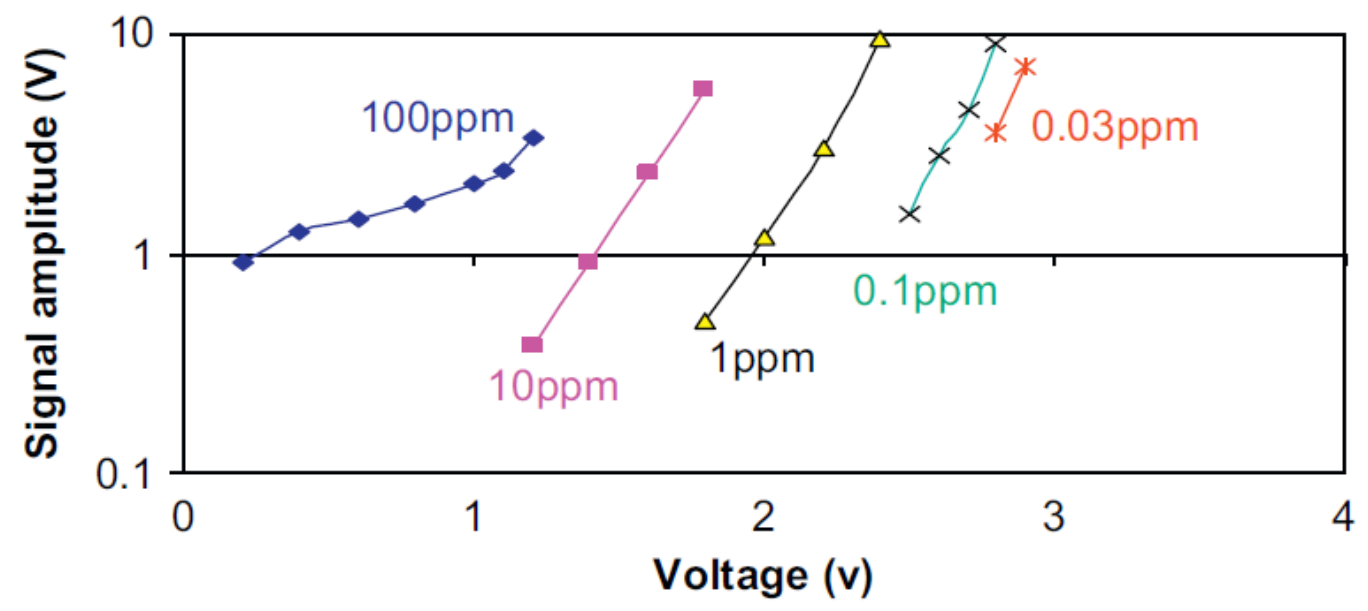

Figure 6: Signal vs. voltage curves for the SWC operating in pulse mode and detecting various concentrations of benzene. At a benzene concentration $c=100$ ppm the measurements were performed either in ionization chamber mode, or at a gain of 3; at c $\sim 0.03$ ppm the gas gain during the measurements was increased to $\sim 4 \times 10^{3}$. 
Nuclear Instruments and Methods in Physics Research Section A: Accelerators, Spectrometers, Detectors and Associated Equipment, 2011, 628(1), 187-189, Proceedings of the $12^{\text {th }}$ International Vienna Conference on Instrumentation,

doi:10.1016/j.nima.2010.06.313

\section{Tables}

Table 1: Minimum detector activities (MDA) in $\mathrm{Bq} / \mathrm{m}^{3}$ of our detectors and of Atmos, measured for different integration times.

\begin{tabular}{|c|c|c|c|}
\hline $\begin{array}{c}\text { Time of } \\
\text { counting (min) }\end{array}$ & SWC MDA & MWPC MDA & Atmos MDA \\
\hline 0.2 & 1300 & 625 & \\
\hline 1 & 420 & 140 & 150 \\
\hline 2 & 270 & 75 & \\
\hline 4 & 175 & 43 & \\
\hline
\end{tabular}

